SCIENTIFIC LETTER

\title{
Calcium score as assessed by multi-slice computed tomography does not predict maximum plaque burden: an in vitro study
}

\author{
M Frick, F Karakolcu, H Gschnitzer, H F Alber, A Stoeger, P Obrist, G Friedrich, F Weidinger, \\ O Pachinger, S P Schwarzacher
}

ए lectron beam computed tomography (EBCT) has been extensively evaluated for the detection of coronary calcification and atherosclerosis. ${ }^{1}$ However, the role of coronary calcium score, measured with EBCT, in predicting the severity of coronary stenosis is still unclear. In some population groups there seems to be a robust association of calcium score and severity of stenosis, ${ }^{2}$ whereas other studies have demonstrated that EBCT calcium score shows only a modest association with coronary stenosis, which may be due to remodelling. ${ }^{3}$

The new generation of multi-slice computed tomography (CT) offers the opportunity to assess coronary calcification with a shorter scanning time and a greater resolution. However, the association of CT assessed coronary calcification and coronary artery plaque burden is unknown. Intravascular ultrasound (IVUS) is the gold standard for the assessment of coronary atherosclerosis. Therefore, the aim of this study was to investigate the association of CT assessed calcium score with IVUS assessed plaque burden.

\section{METHODS}

Thirty two coronary arteries (22 right coronary arteries, five left anterior descending coronary arteries, and five circumflex arteries) were obtained from 26 necropsies within 24 hours of death, from people aged between 53-96 years. The investigations conformed to the principles outlined in the Declaration of Helsinki.

After harvesting, the coronary arteries were elastically mounted in a special container filled with physiologic saline solution. Thereafter an IVUS catheter (40 MHz, Atlantis SR Plus, Boston Scientific International, France) was introduced into the lumen and an automated pullback $(0.5 \mathrm{~mm} / \mathrm{s})$ was performed. Calcified lesions were identified by their echogenicity and their acoustic shadow. Vessel area and lumen area were measured and plaque area and plaque burden were calculated. Mean plaque burden was defined as the sum of plaque burden from the whole artery divided by the number of measurements; maximum plaque burden was the greatest plaque burden of the vessel. Next, the container was placed into the CT (Somatom Plus Volume Zoom 4, Siemens) and a standardised $3 \mathrm{~mm}$ thick slice protocol was performed. Coronary calcification was said to be present when at least one voxel within the arterial cross section reached more than 130 Hounsfield units. Calcium score was quantified using the Agatston score. Finally, histological analyses of slices were performed in segments where IVUS had detected coronary calcification but CT had not. Calcification was defined by typical basophile colouration after haematoxylin and eosin colouration.

Data are expressed as mean (SD) and percentage. Linear correlation between calcifications as assessed by CT and IVUS methods were calculated using Spearmen's correlation

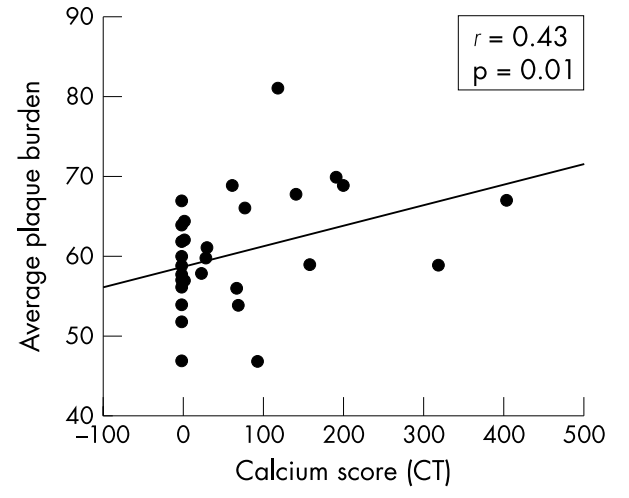

Figure 1 (A) Association of maximum plaque burden, and (B) average plaque burden with calcium score on an artery basis.

coefficient. Differences between groups were analysed using non-paired Student's $t$ test. A probability value of $\mathrm{p}<0.05$ was considered significant.

\section{RESULTS}

CT detected a positive calcium score in $66 \%$ of arteries ( 21 of 32 arteries) and in $24 \%$ of segments (141 of 599 segments). IVUS revealed calcification in $75 \%$ of arteries ( 24 of 32 arteries) and in 34\% of segments (205 of 599 segments), resulting in a sensitivity of CT for detecting coronary calcification of $88 \%$ (entire artery) and $71 \%$ (single segments) compared to IVUS. Histological analyses of the segments in which IVUS, but not CT, had detected coronary calcification, confirmed the presence of calcification. Linear regression analyses showed a close correlation between calcium score as assessed by CT and calcification as measured with IVUS $(r=0.89, \mathrm{p}<0.001)$.

No significant differences in average (62.1 (7.3) $v 57.3$ (5.2), $\mathrm{p}=0.06$ ) and maximum plaque burden (73.4 (7.0) $\mathrm{v}$ $74.7(10.1), p=0.33$ ) were found when entire arteries with a positive CT assessed calcium score were compared to arteries with no calcification. Univariate analyses showed no association between maximum plaque burden and CT assessed calcium score $(r=0.07$, not significant), but revealed a significant correlation between average plaque burden and CT assessed calcium score (fig 1). Also, when single segments were compared, plaque burden was significantly higher in segments with a positive calcium score compared to segments with no calcification as assessed by CT

Abbreviations: $C T$, computed tomography; $\mathrm{EBCT}$, electron beam computed tomography; IVUS, intravascular ultrasound 
(66.0 (9.5) v $59.6(9.1), \mathrm{p}<0.01)$. In addition, plaque burden detected by IVUS correlated with CT detected calcium score on a single segment basis $(r=0.30, \mathrm{p}<0.001)$.

\section{DISCUSSION}

This study provides evidence for three important findings. Firstly, CT can accurately detect coronary calcification; secondly, average and maximum plaque burden are not different between CT detected calcium positive and negative arteries; and thirdly, a significant, although weak correlation between average plaque burden and calcium score was found on a single segment basis.

The role of EBCT in predicting the severity of coronary artery stenosis is controversial and it has been demonstrated that the negative predictive value of EBCT is very high. ${ }^{2}{ }^{4}$ We have seen no significant difference in average and maximum plaque burden (as assessed by IVUS) between CT assessed calcium positive and negative arteries. Consequently, our study suggests that calcium score as assessed by CT may not be a reliable tool to discriminate between patients with and patients without coronary stenosis. Furthermore, as the arteries without calcification showed a similar maximum plaque burden compared to $\mathrm{CT}$ assessed calcium positive vessels, the negative predictive value, at least in our study, is very low. This result is discrepant to earlier studies which may be due to different patient populations. ${ }^{45}$ Earlier studies have mainly examined highly selected patients, while our patient population more accurately reflects a general population. Consequently, our data do not support the use of a negative calcium score as an exclusion criterion for the presence of coronary artery disease.

On univariate analyses calcium score correlated with average plaque burden supporting an association of coronary calcification with the extent, but not necessarily with the severity, of the disease. This is in accordance to previous studies that have found a high positive predictive value of coronary calcification for the presence of atherosclerosis. ${ }^{2} 5$
The association of coronary calcification with the extent of the disease seems to confirm that CT screening may provide better information on risk of events than on stenosis.

Although we have investigated 32 coronary arteries our sample size is still small and we did not investigate all main coronary arteries from all patients. We were therefore not able to analyse data on a heart by heart basis; however, we suggest this approach would probably not have changed the results given the aim of this study.

\section{Authors' affiliations}

M Frick, F Karakolcu, H Gschnitzer, H F Alber, G Friedrich, F Weidinger, O Pachinger, S P Schwarzacher, Division of Cardiology, Department of Internal Medicine, University of Innsbruck, Austria

A Stoeger, Department of Radiology, University of Innsbruck

P Obrist, Institute of Pathology, University of Innsbruck

Correspondence to: Dr Severin Schwarzacher, Division of Cardiology, Department of Medicine, University of Innsbruck, Anichstrasse 35, A-6020 Innsbruck, Austria; severin77@aon.at

Accepted 27 October 2003

\section{REFERENCES}

1 Gutfinger DE, Leung $C Y$, Hiro $T$, et al. In vitro atherosclerotic plaque and calcium quantitation by intravascular ultrasound and electron-beam computed tomography. Am Heart J 1996;131:899-906.

2 Bielak LF, Rumberger JA, Sheedy PF 2nd, et al. Probabilistic model for prediction of angiographically defined obstructive coronary artery disease using electron beam computed tomography calcium score strata. Circulation 2000;102:380-5

3 Detrano R, Hsiai T, Wang S, et al. Prognostic value of coronary calcification and angiographic stenoses in patients undergoing coronary angiography. J Am Coll Cardiol 1996;27:285-90.

4 Guerci AD, Spadaro LA, Popma JJ, et al. Relation of coronary calcium score by electron beam computed tomography to arteriographic findings in asymptomatic and symptomatic adults. Am J Cardiol 1997;79:128-33.

5 Rumberger JA, Sheedy PF, Breen JF, et al. Electron beam computed tomographic coronary calcium score cutpoints and severity of associated angiographic lumen stenosis. J Am Coll Cardiol 1997;29:1542-8.

\section{IMAGES IN CARDIOLOGY}

\section{Postoperative hydropneumopericardium}

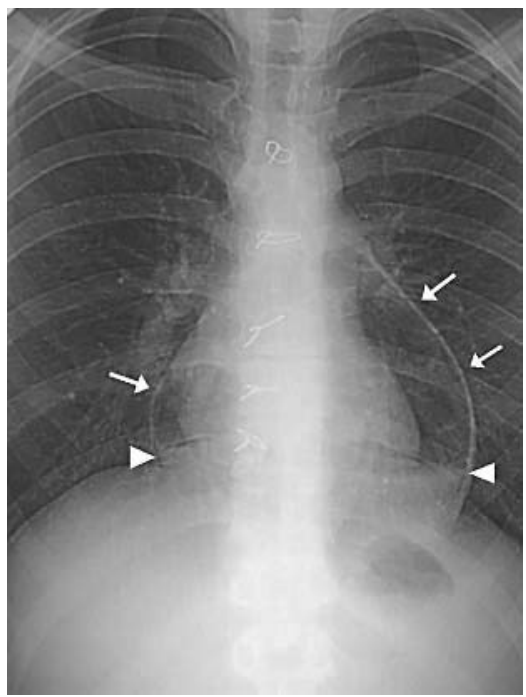

Chest $x$ ray of the pericardium showing trapped air (arrows) and effusion level (arrowheads).
20 year old man with a sinus venosus atrial septal defect underwent surgical correction with a pericardial baffle; the superior vena cava was not patched. Seven days postoperatively he developed pericardial effusion, which was initially treated with nonsteroidal anti-inflammatory drugs. The pericardium was subsequently drained surgically, through a subxyphoid approach, after an unsuccessful percutaneous attempt. A pericardial drain was inserted the day after the procedure because of a hydropneumopericardium, revealed by standard chest $x$ ray (left). We hypothesise that air trapping in the pericardium occurred because of a valve mechanism caused by the anti-Trendelenburg position.

E Aidala

A Valori

P A Abbruzzese enri.ema@tiscalinet.it 